\title{
Neuroprotective Effects of Lycium Barbarum Berry on Neurobehavioral Changes and Neuronal Loss in the Hippocampus of Mice Exposed to Acute Ionizing Radiation
}

Lei Guo

Yangtze University

Qian-Qian Du

Yangtze University

Piao-Qin Cheng

Yangtze University

Ting-Ting Yang

Yangtze University

Chao-Qun Xing

Yangtze University

Xue-Zhi Luo

Yangtze University

Xiao-Chun Peng

Yangtze University

Feng Qian (D 49605795@qq.com )

Yangtze University https://orcid.org/0000-0001-5766-4623

Jiang-Rong Huang

Yangtze University

Feng-Ru Tang

National University of Singapore

Research

Keywords: lycium barbarum berry, ionizing radiation, cognitive dysfunction, calbindin, parvalbumin

Posted Date: July 13th, 2021

DOl: https://doi.org/10.21203/rs.3.rs-695235/v1

License: (c) (1) This work is licensed under a Creative Commons Attribution 4.0 International License.

Read Full License 
Version of Record: A version of this preprint was published at Dose-Response on October 1st, 2021. See the published version at https://doi.org/10.1177/15593258211057768. 


\section{Neuroprotective effects of lycium barbarum berry on}

\section{neurobehavioral changes and neuronal loss in the hippocampus of mice exposed to acute ionizing radiation}

Lei Guo ${ }^{1 \#}$, Qian-Qian Du ${ }^{1 \#}$, Piao-Qin Cheng ${ }^{1 \#}$, Ting-Ting Yang ${ }^{2}$, Chao-Qun Xing ${ }^{1}$, Xue-Zhi Luo ${ }^{1}$, Xiao-Chun Peng ${ }^{3}$, Feng Qian ${ }^{2 *}$, Jiang-Rong Huang ${ }^{1 *}$, Feng-Ru Tang ${ }^{4 *}$

${ }^{1}$ Department of Traditional Chinese Medicine, Health Science Center, Yangtze University, Jingzhou, Hubei Province, China, 434023

${ }^{2}$ Department of Physiology, School of Basic Medicine, Health Science Center, Yangtze University, Jingzhou, Hubei Province, China, 434023

${ }^{3}$ Department of Pathophysiology, School of Basic Medicine, Health Science Center, Yangtze University, Jingzhou, Hubei Province, China, 434023

${ }^{4}$ Radiation Physiology Laboratory, Singapore Nuclear Research and Safety Initiative, National University of Singapore, Singapore

\#These three undergraduate students worked as a team and contributed equally to this study.

\section{*Correspondence Authors:}

Dr. Feng Qian, Department of Physiology, School of Basic Medicine, Health Science Center, Yangtze University, Jingzhou, Hubei Province, China, 434023.

E-mail: 49605795@qq.com, Tel.: +8618986655233

Dr. Jiang-Rong Huang, Department of Traditional Chinese Medicine, Health Science Center, Yangtze University, Jingzhou, Hubei Province, China, 434023.

E-mail: huangjr999@ sina.com, Tel.: +8613607216868

Dr. Feng-Ru Tang, Radiation Physiology Laboratory, Singapore Nuclear Research and Safety Initiative, National University of Singapore, Singapore. E-mail: tangfr@gmail.com, Tel.: +65 66011094, Fax: +65 68726840 


\begin{abstract}
Background: Brain exposure to ionizing radiation during the radiotherapy of brain tumor or metastasis of peripheral cancer cells to the brain has resulted in cognitive dysfunction by reducing neurogenesis in hippocampus.

Methods: To demonstrate the radioprotective effect of lycium barbarum berry extract (Lyc), we evaluated the neurobehavioral alterations and the numbers of NeuN, calbindin (CB) and parvalbumin (PV) immunopositive hippocampal neurons in BALB/c mice after acute 5.5 Gy radiation with/without oral administration of Lyc at the dosage of $10 \mathrm{~g} / \mathrm{kg}$ daily for 4 weeks.

Results: The results showed that Lyc could improve irradiation-induced animal weight loss, depressive behaviors, spatial memory impairment and hippocampal neuron loss. Immunohistochemistry study demonstrated that the loss of NeuN-immunopositive neuron in the hilus of the dentate gyrus, CB-immunopositive neuron in CA1 strata radiatum, lacunosum moleculare and oriens, and PV-positive neuron in CA1 stratum pyramidum and stratum granulosum of the dentate gyrus after irradiation were significantly improved by Lyc treatment.
\end{abstract}

Conclusions: The neuroprotective effect of Lyc on those hippocampal neurons may benefit the configuration of learning related neuronal networks and then improve radiation induced neurobehavioral changes such as cognitive impairment and depression. It suggests that lycium barbarum berry may be used as a potential radio-neuro-protective food supplement to prevent ionizing radiation induced neuron loss and neuropsychological disorders.

Keywords: lycium barbarum berry, ionizing radiation, cognitive dysfunction, calbindin, parvalbumin 


\section{Introduction}

Ionizing radiation has been widely applied to brain disease diagnosis and radiotherapy to treat brain tumor and to prophylactically prevent metastasis of peripheral cancer cells to the brain. However, acute or fractional accumulative ionizing radiation may result in neurodegenerative disorders including cognitive dysfunction, sensory disturbance and poor motor coordination(Jacob et al., 2018). Previous studies have shown that irradiation could lead to cognitive dysfunction by reducing neurogenesis in hippocampus after the acute radiation exposure (Guo et al., 2019; Hladik and Tapio, 2016; Tang et al., 2017; Wang et al., 2019b). Although many neuroprotective agents have been reported to have significant efficacy on radiation-induced cognitive dysfunction, amifostine is still the only approved radioprotectant during radiotherapy(Allen et al., 2018). Our previous study have indicated that the oral administration of Chinese herbal medicine (CHM) epimedium after acute radiation exposure could significantly improve the hippocampal neurogenesis and radiation-induced cognitive dysfunction in BALB/c mice(Wang et al., 2019b). This implicated that CHMs may have great advantages as radio-neuro-protectants because of their anti-oxidative and immunoregulatory effects.

Lycium barbarum, also named Goji berry and wolfberry, which is rich of lycium barbarum polysaccharides (LBPs), has been used as food supplement and herbal medicine in China for thousands of years and has been increasingly accepted as anti-oxidative and anti-aging fruit in Western countries(Ma et al., 2019; Ulbricht et al., 2014). Its active ingredient LBPs can improve retinal function and reduce retinal neuron damage(Li et al., 2019; Yang et al., 2017), reduce blood lipid and glucose(de Souza Zanchet et al., 2017; Wang et al., 2019a), boost immune system to anti-allergy and anti-cancer(Ulbricht et al., 2014), protect against radiotherapy or chemotherapy induced tissue damages(Tian et al., 2019), prevent neuron loss and enhance neurogenesis(Zhou et al., 2020). As a nutrient rich “superfood”, lycium barbarum berry can be used as a daily diet without serious accumulative toxins or 
side-effect(Ma et al., 2019). Its potential neuroprotective effects during and after radiotherapy of brain tumor should be evaluated. In the present study, we studied the radio-neuro-protective effects of lycium barbarum berry extract on neurobehavioral changes and hippocampal neuron loss in the mouse model of acute ionizing radiation exposure.

\section{Materials and methods}

\subsection{Lycium barbarum berry extraction}

Five hundred gram dried lycium barbarum berries (Zhongning, Ningxia, China) were crushed and decocted in $3 \mathrm{~L}$ boiled distilled water for 2 hours. The decocted liquid was filtrated and the residue was decocted again in $2 \mathrm{~L}$ water. The two filtrates were combined and concentrated it in rotary evaporator under $60^{\circ} \mathrm{C}$ to obtain $350 \mathrm{~g}$ thick paste which was stored under $4^{\circ} \mathrm{C}$.

\subsection{Experimental animal}

Male specific-pathogen-free (SPF) BALB/c mice were purchased from Hubei experimental animal research center licensed with the number SCXK (Hubei) 2015-0018, and accommodated in local animal room to 8-week-old and body weight $22 \pm 2 \mathrm{~g}$ before irradiation. Animals were divided into four groups: 1) sham X-ray exposure group (Sham) of 10 mice with oral saline administration after sham exposure; 2) experimental control group (Exp-Ctrl) of 10 mice with 5.5 Gy X-ray whole body irradiation followed by oral saline administration; 3 ) positive experimental control group (Ami-Exp) of 10 mice with the amifostine pretreatment (at $100 \mathrm{mg} / \mathrm{kg}$, batch number: J0311A, Dalian Meilun Biotech. Co., China) at $30 \mathrm{~min}$ before 5.5 Gy X-ray whole body irradiation; 4) experimental group (Exp-Lyc) of 10 mice with 5.5 Gy X-ray whole body irradiation followed by the oral administration of the lycium barbarum berry extract ( $10 \mathrm{~g} / \mathrm{kg}$ per day) $2 \mathrm{~h}$ after radiation exposure on the first day, and the lycium barbarum berry extract treatment was continued for 4 weeks. The body weight was recorded after the irradiation. Behavioral tests were 
conducted four weeks after radiation exposure and the mice were then sacrificed for the brain sample collection.

\subsection{Behavioral tests}

To test if irradiation induced depression and spatial memory impairment and if Lyc could improve those changes, open field, Tail suspension, forced swimming and Morris water maze tests were used. Behavioral tests were conducted in a quiet room with dim light and constant temperature $\left(20-25^{\circ} \mathrm{C}\right)$ using SuperMaze animal behavior record and analysis system (Shanghai Xinruan InfoTech Co., China). Tail suspension and forced swimming immobility times were recorded for 4 min after $2 \mathrm{~min}$ environmental adaptation. The open field size of $50 \mathrm{~cm}$ (length) $\times 50 \mathrm{~cm}$ (width) $\times 30$ $\mathrm{cm}$ (height) was divided into 25 boxes to record the total movement distance and the time spent in the central area (central 9 boxes) for $10 \mathrm{~min}$. The Morris water maze experiments were done as described previously(Wang et al., 2019b). The hidden platform escape latency was recorded for 5 days. On the day 6 , after removing the platform, the times that the mice passed though platform area and the total resident time that the mice spent in the platform quadrant in $60 \mathrm{~s}$ were recorded.

\subsection{Immunohistochemistry}

The mice were anesthetized with $1 \%$ pentobarbital and fixed with $4 \%$ paraformaldehyde through transcardial perfusion after behavioral tests. The brain was removed, postfixed with the same fixative overnight and then stored in 30\% sucrose solution prepared with $0.1 \mathrm{M}$ phosphate buffer (pH: 7.4). For immunohistochemistry, sagittal brain sections with $40 \mu \mathrm{m}$ thickness were cut and a set of three serial sections was prepared and sections collected were placed individually in different wells of a 24 well tissue culture dish for the control, NeuN, calbindin (CB), and parvalbumin (PV) immunohistochemical reaction. The freely floating sections were treated in $4 \%$ normal goat serum for $2 \mathrm{~h}$ at room temperature. All sections were then washed in $0.1 \mathrm{M}$ phosphate-buffered saline (PBS) containing NeuN (1:100) (Invitrogen, USA), CB (1: 2000) and PV (1:1000) (Swant, Switzerland). 
After incubation, sections were washed in PBS and placed for $1 \mathrm{~h}$ in biotinylated goat anti-rabbit IgG (Vector Laboratories, Burlingame, CA, USA) diluted 1:2000 in PBS/Triton X-100. After two washes in PBS, they were placed in avidin- biotin complex (ABC) reagent (Vector Laboratories) in PBS/Triton X-100 for $1 \mathrm{~h}$, washed in PBS and reacted in a solution of $0.12 \% \mathrm{H} 2 \mathrm{O} 2$ and $0.05 \%$ 3,3-diaminobenzidine (DAB) (Sigma, USA) in Tris buffer (TB) for $15 \mathrm{~min}$, then mounted, dehydrated, coverslipped and photographed by using image analysis system.

\subsection{Cell counting and statistical analysis}

NeuN immunopositive neurons in the hilus of the dentate gyrus, CB immunopositive interneurons in the area of CA1 strata radiatum lacunosum moleculare and stratum oriens, PV immunopositive interneurons in the CA1 stratum pyramidum and the stratum granulosum of the dentate gyrus in the dorsal hippocampus were counted by using Leica DM4 B upright microscope and LAS X software (Leica Microsystems, Jermany). The sagittal brain sections from 5 mice in each group were used for immunopositive cell counting. One- way ANOVA followed by the Student's t-test were used for statistical analysis and statistical significance was considered when $\mathrm{P}<$ 0.05 . All the data were showed as mean \pm SD.

\section{Results}

\subsection{Body weight}

X-ray irradiation with 5.5Gy (Exp-Ctrl) induced a significant body weight loss in the first week after exposure compared to the sham radiation exposure mice (Sham) (Fig. 1). Oral administration of lycium barbarum berry extract at the dosage of $10 \mathrm{~g} / \mathrm{kg}$ per day (350 g extract paste from $500 \mathrm{~g}$ dried lycium barbarum berries) (Exp-Lyc) prevented animal weight loss from the second to third week after irradiation, similar to amifostine pretreatment (Ami-Exp) with intraperitoneal injection at $100 \mathrm{mg} / \mathrm{kg} 30$ min before irradiation (Fig. 1). Both therapeutic strategies in Exp-Lyc and Ami-Exp groups effectively improved the animal weight gain to the normal level in the Sham 
group by the end of the fifth week after irradiation. At this time point, animals in Exp-Lyc and Ami-Exp groups were significantly heavier than those in Exp-Ctrl $(\mathrm{P}<$ 0.05), but had no significant weight difference compared to the Sham group.

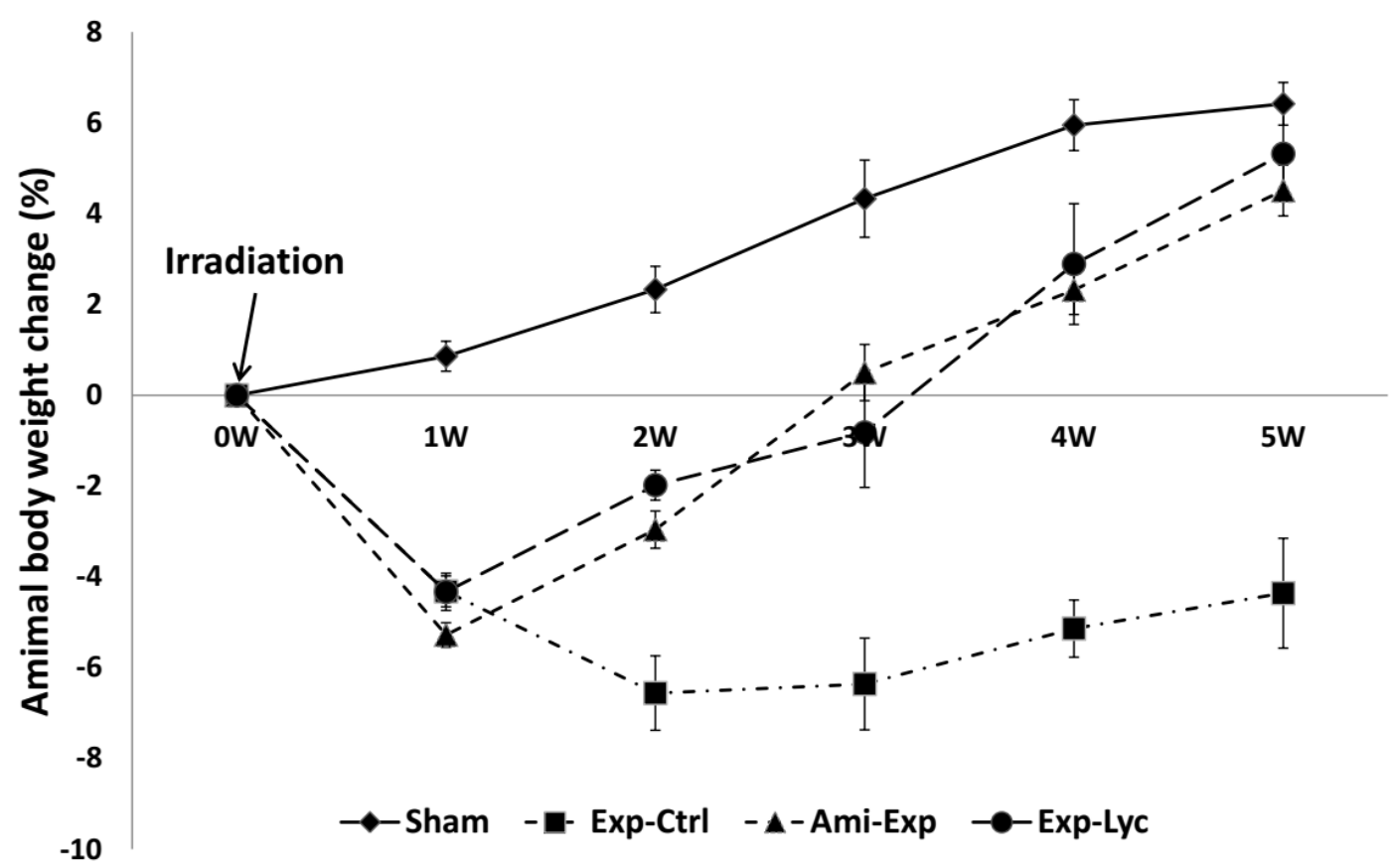

Figure 1. Effect of lycium barbarum berry extract on the body weight of radiation exposed

BALB/c mice. The body weight of the mice in the sham exposure group (Sham) increases steadily, but it decreases significantly after acute X-ray irradiation with 5.5Gy in the experimental control (Exp-Ctrl), mice treated with lycium barbarum berries (Exp-Lyc) and amifostine (Ami-Exp) at 1 week after the exposure ( $\mathrm{P}<0.05$ compared to the Sham group). From the second week, amifostine pretreatment (Ami- Exp) and oral lycium barbarum berry extract treatment after exposure (Exp-Lyc) reverse the animal weight loss and these animals have similar body weight to Sham group at the end of the fifth week $(\mathrm{P}>0.05)$. However, Exp-Ctrl group has a significant weight loss from 1 to 5 week(s) after radiation exposure when compared to the other three groups ( $\mathrm{P}<0.05, \mathrm{n}=10$ in each group).

\subsection{Behavioral tests}

The average tail suspension (Fig. 2A) and forced swimming (Fig. 2B) immobility time in 2 min of those radiation exposed mice with saline treatment were significantly 
longer than the sham exposed mice. Amifostine or lycium barbarum berry treatment significantly reduced immobility time when compared to irradiated animals treated with saline (Fig. 2A,B). No significant difference in immobility time was observed among those exposed animals treated with amifostine or lycium barbarum berry and the Sham group. Open field experiment showed that the total travel distance in the Exp-Ctrl group within 10 min was less than the Sham group, amifostine or lycium barbarum treated mice, indicating both treatments significantly improved animal locomotor activity (Fig $2 \mathrm{C}, \mathrm{P}<0.05$ ). Similarly, lycium barbarum berry also reduced the time animals stayed in the central area of test field when compared to radiation exposed control mice (Fig 2D, $\mathrm{P}<0.05$ ). Morris water maze experiments showed that the average platform escape latency time of radiation exposure mice was significantly longer than the Sham group on the day 5, (Fig 3A, B, P < 0.05). In addition, the average platform crossing time and platform quadrant resident time of the Exp-Ctrl group on day 6 were less than the Sham group (Fig 3C, P < 0.05). Amifostine or lycium barbarum berry treatment reduced the escape latency and increased the platform crossing time and platform quadrant resident time significantly, indicating that both treatments could improve the spatial memory of mice with radiation exposure. These data of behavioral tests demonstrated that the oral administration of lycium barbarum berry extract could effectively prevent or treat the radiation damages in the motor activity and cognitive function of mice. 

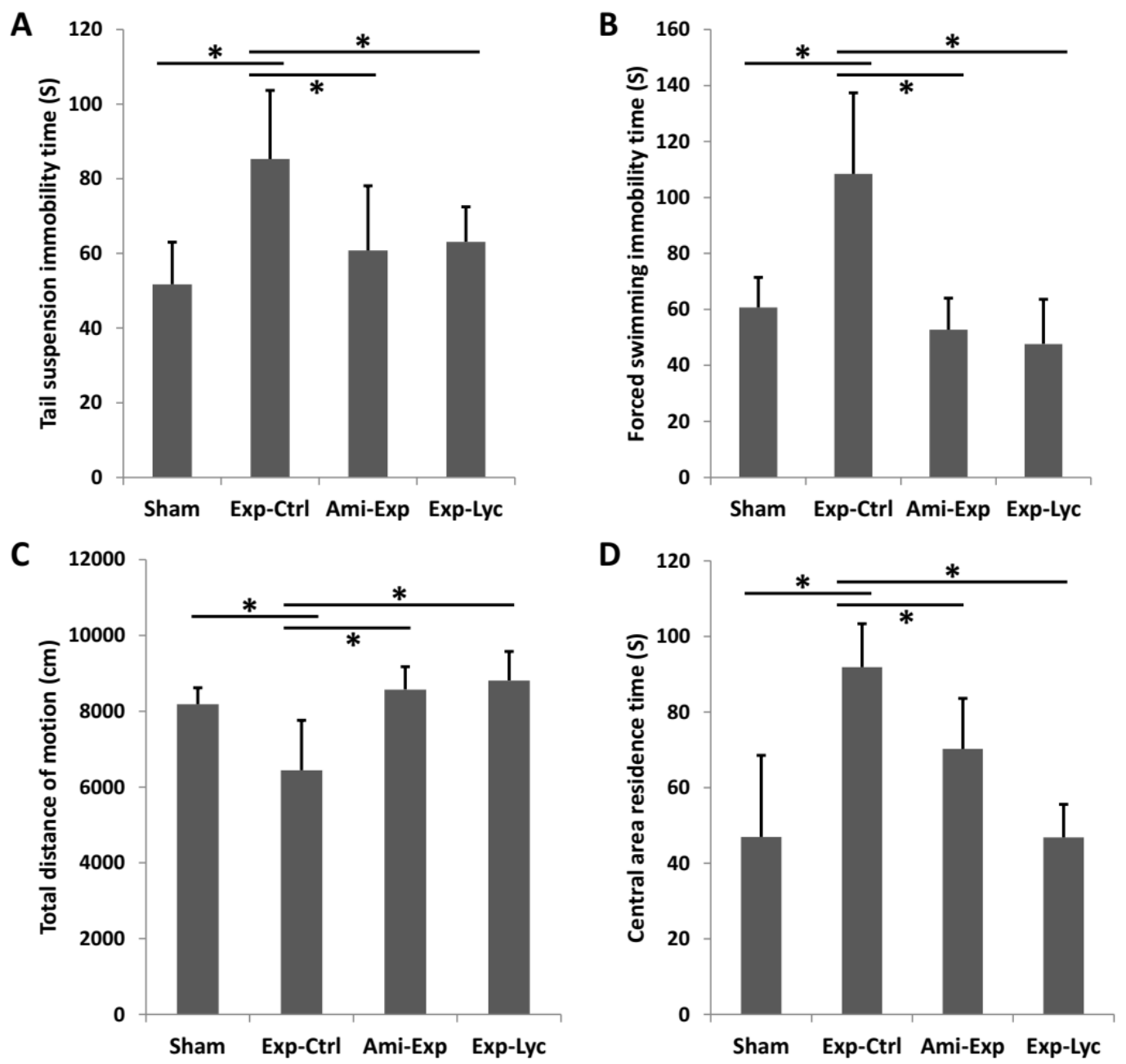

Figure 2. Tail suspension, forced swimming and open field tests. Similar to the amifostine pretreated (Ami-Exp) group, lycium barbarum berry extract (Exp-Lyc) significantly reduces the immobility times in tail suspension (A) and forced swimming (B) tests. Open field test shows the average total travelling distance of the mice in radiation exposure control group (Exp-Ctrl) is significantly less than those of the mice in the other three groups (C). However, the average central area staying time of Exp-Ctrl mice is significantly longer (D). Asterisks * indicate $\mathrm{P}<0.05$. The values are the mean \pm SD calculated from the data of 10 mice in each group. 


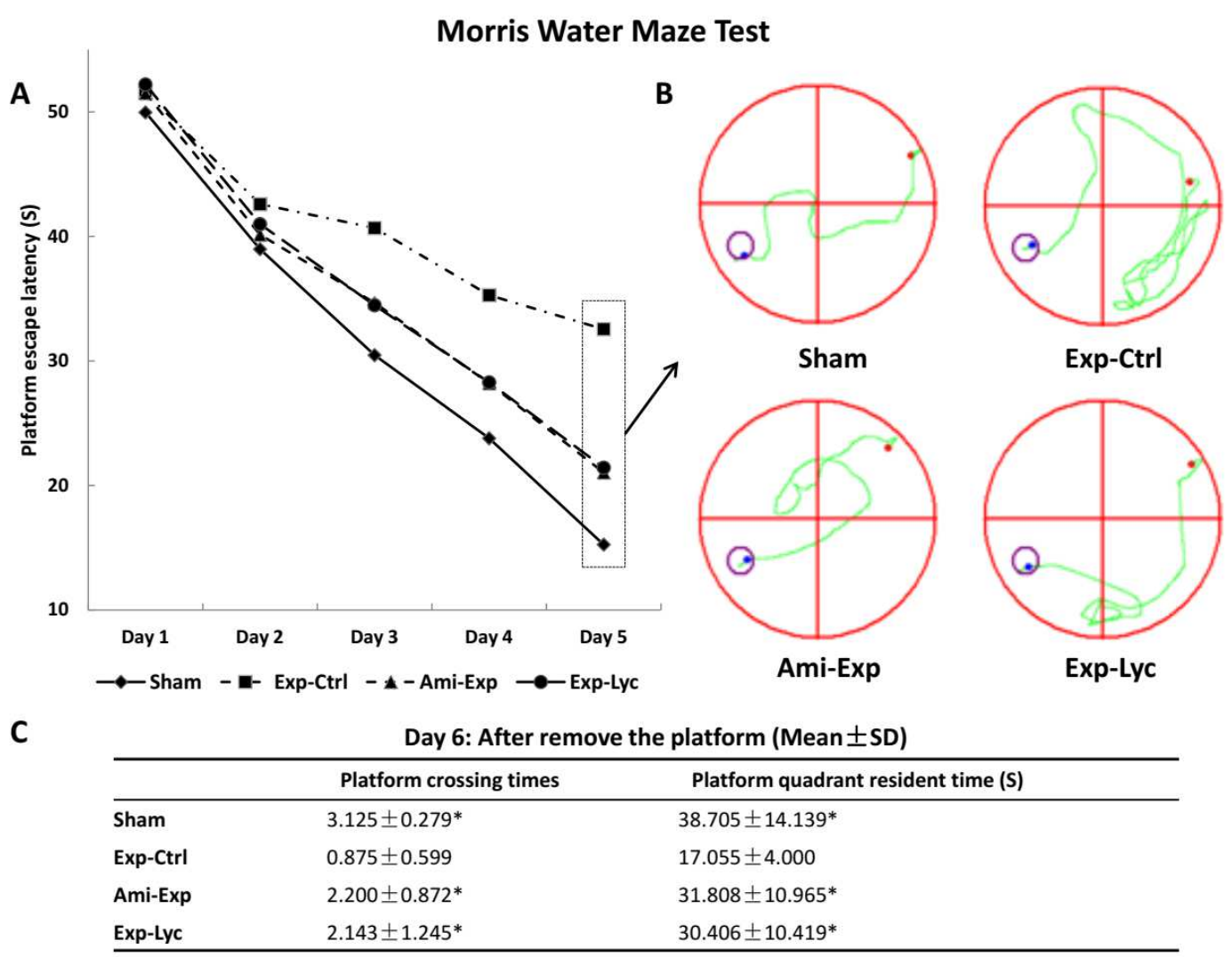

Figure 3. Morris water maze test. Morris water maze test shows that the average escape latency of the Exp-Ctrl group is significantly longer than those of the Sham group, The Ami-Exp or Exp-Lyc group in the training trials from the day 3 to day $5(\mathrm{P}<0.05, \mathrm{~A}, \mathrm{~B})$. The average time spent in the platform quadrant and the times of animal crossing platform area of the Exp-Ctrl group are significantly lower than those of other three groups on day $6(\mathrm{P}<0.05, \mathrm{C})$. Asterisks * in panel $\mathrm{C}$ indicate $\mathrm{P}<0.05$ when compared with the Exp-Ctrl group. The values are the mean \pm SD calculated from the data of 10 mice in each group.

\subsection{Immunohistochemistry}

NeuN, CB and PV immunopositive neurons in the target areas of dorsal hippocampus were counted from 5 slices of each mouse brain. The number of NeuN immunopositive neurons in the hilus of the dentate gyrus was significantly reduced after irradiation with 5.5 Gy (Fig 4, Exp-Ctrl compared to Sham, P < 0.05). Both amifostine pretreatment and lycium barbarum berry extract treatment prevented hilar neuronal loss when compared to the Exp-Ctrl (Fig 4, Ami-Exp or Exp-Lyc compared to Exp-Ctrl, $\mathrm{P}<0.05)$. Similarly, the number of $\mathrm{CB}$ positive neurons in the strata 
radiatum lacunosum moleculare and stratum oriens, and the number of PV positive interneurons in the CA1 stratum pyramidum and the stratum granulosum of the dentate gyrus were also reduced by radiation exposure (Fig 5, 6, P < 0.05), indicating the radiation induced neuron loss in these areas. Oral administration of lycium barbarum berry effectively increased the numbers of CB and PV immunopositive cells in the corresponding areas (Fig 5, 6, P < 0.05). These immunochemistry results demonstrated that the lycium barbarum berry could effectively prevent the loss of NeuN, CB and PV immunopositive cells in the hilus and stratum granulosum of the dentate gyrus, strata radiatum lacunosum moleculare, stratum oriens and pyramidum of CA1 area of the mouse hippocampus. 

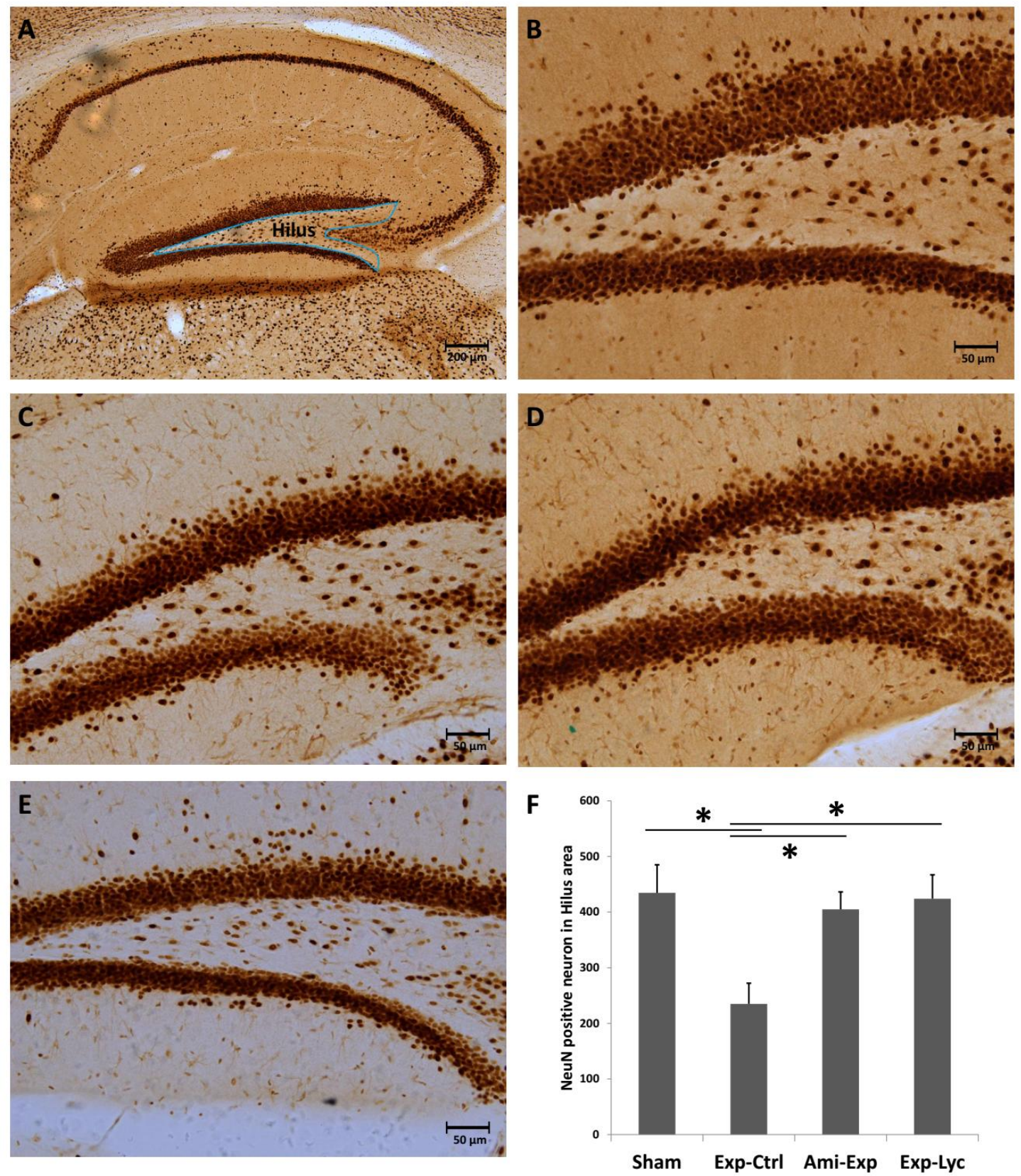

Figure 4. NeuN immunohistochemistry. A, NeuN immunohistochemical staining shows neurons in the hilus of the dentate gyrus (the triangle area indicated in the slice). B, C, D and E show the NeuN immunopositive neurons in the hilus of the dentate gyrus of the representative slices from the Sham, Exp-Ctrl, Ami-Exp and Exp-Lyc groups respectively. F, Statistical analysis shows the number of NeuN immunopositive neurons in the hilus of the Exp-Ctrl group (pyramidal neurons in CA3c were excluded) is significantly fewer than the Sham group. The Ami-Exp or Exp-Lyc group has more NeuN immunopositive neurons in the hilus than the Exp-Ctrl group. Asterisks * indicate $\mathrm{P}<0.05$ when compared with the Exp-Ctrl group ( $\mathrm{n}=5$ in each group, cell number is indicated as the mean $\pm \mathrm{SD}$ ). 

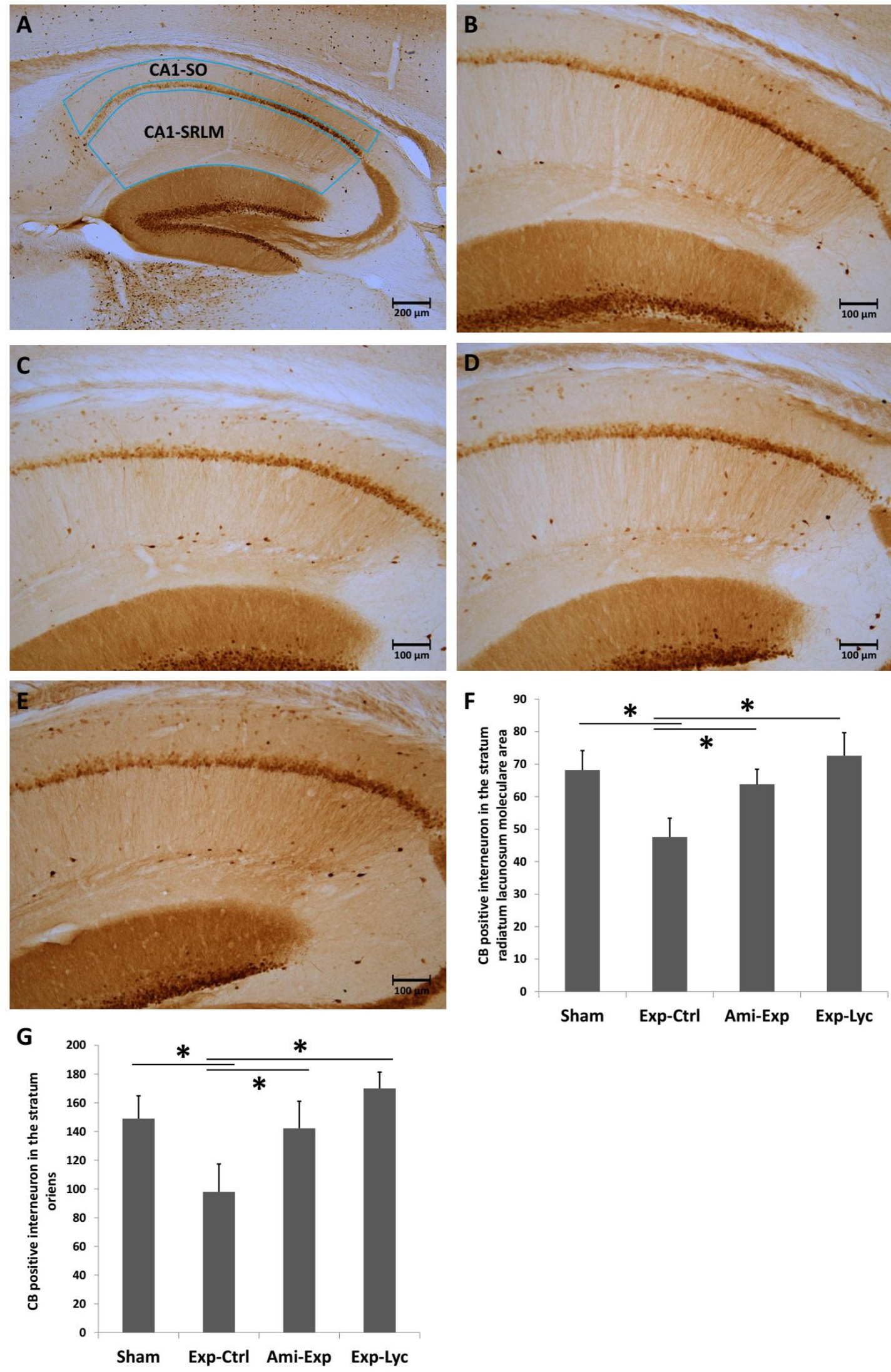
Figure 5. Calbindin (CB) immunohistochemistry. A, CB immunohistochemical staining shows interneurons in the strata radiatum, lacunosum moleculare (SRLM) and oriens (SO) in CA1 area (the areas indicated in the slice). B, C, D and $\mathrm{E}$ show the $\mathrm{CB}$ immunopositive interneurons in the CA1-SRLM and CA1-SO areas of the representative slices from the Sham, Exp-Ctrl, Ami-Exp and Exp-Lyc groups respectively. F and $\mathrm{G}$ show the numbers of $\mathrm{CB}$ immunopositive interneurons in the CA1-SRLM and CA1-SO areas of the Exp-Ctrl group are statistical significantly fewer than the Sham group. Both Ami-Exp and Exp-Lyc groups have more CB immunopositive interneurons in these areas than the Exp-Ctrl group. Asterisks * indicate $\mathrm{P}<0.05$ when compared with the Exp-Ctrl group( $\mathrm{n}=5$ in each group, cell number is indicated as the mean $\pm \mathrm{SD}$ ). 

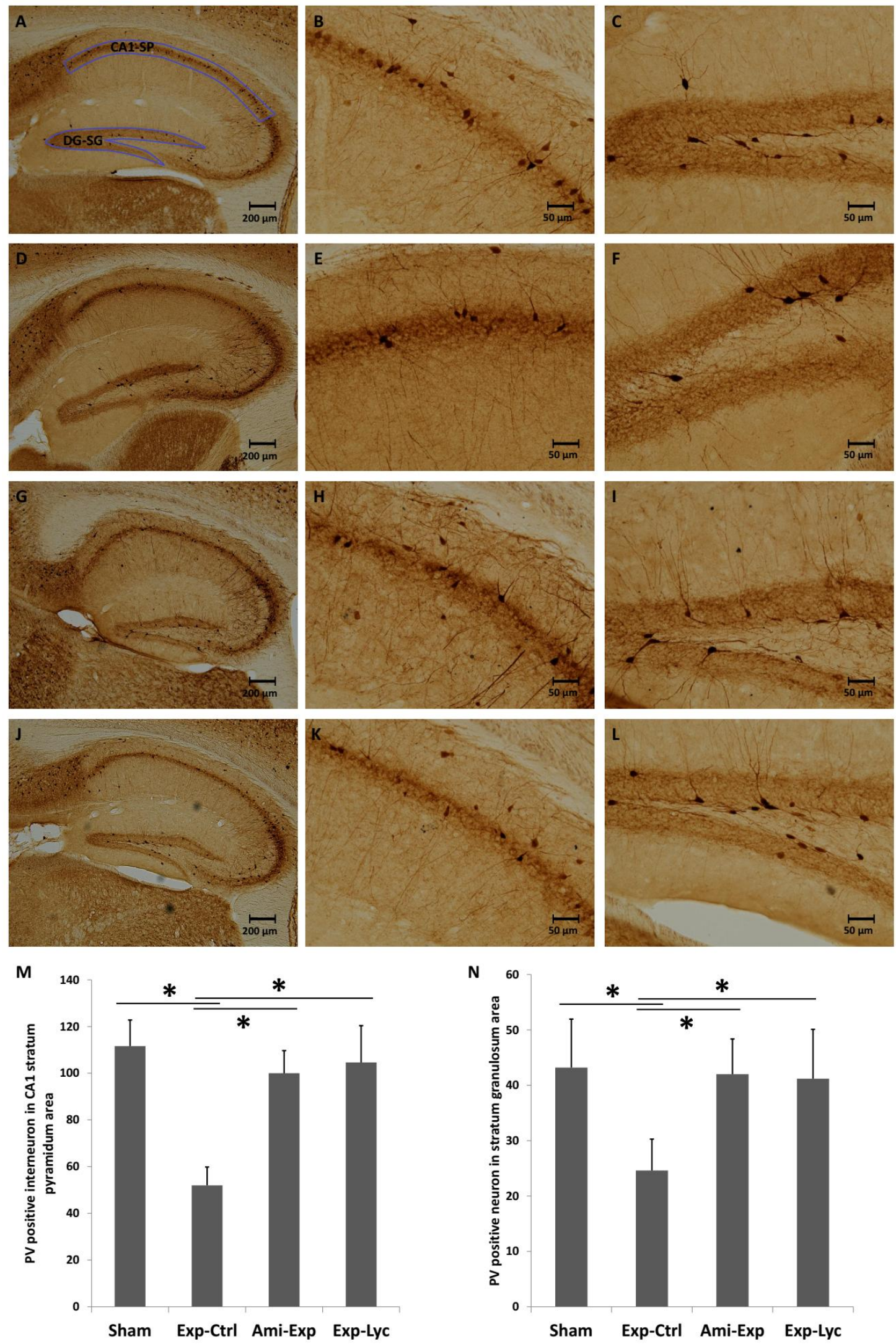

Figure 6. Parvalbumin (PV) immunohistochemistry. The areas indicated in the slice A show the PV immunostaining interneurons in the CA1 stratum pyramidum (CA1-SP, B) and the stratum 
granulosum of the dentate gyrus (DG-SG, C) of the Sham group. The picture D shows significant reduced numbers of PV immunopositive interneurons in the CA1-SP (E, M) and the DG-SG (F, N) of the Exp-Ctrl group when compared to the Sham group. The numbers of PV immunopositive interneurons in the CA1-SP (M) and the DG-SG (N) of the Ami-Exp (G, H, I) or Exp-Lyc group $(\mathrm{J}, \mathrm{K}, \mathrm{L})$ are significantly more than the Exp-Ctrl group. Asterisks * indicate $\mathrm{P}<0.05$ when compared with Exp-Ctrl group ( $\mathrm{n}=5$ in each group, cell number is indicated as the mean $\pm \mathrm{SD}$ ).

\section{Discussion}

\section{Lyc improves radiation-induced body weight loss}

Radiotherapy with ionizing radiation may lead to body weight loss in brain or neck cancer patients(Lee et al., 2019) and animals exposed to acute high dose radiation(Wang et al., 2019b). Lycium barbarum berry has been increasingly accepted as an anti-oxidative, immune-boosting and neuroprotective food supplement which may strengthen the body resistance to damages, pathogens and even ageing(Ma et al., 2019). In the present study, oral administration of Lyc effectively prevented weight loss of the mice X-ray-irradiated with $5.5 \mathrm{~Gy}$, indicating that this traditional herbal medicine should have a role in controlling radiation damages. To further study the radioprotective role of Lyc, we investigated its effect on the neurobehavioral performance and neuroprotection on the hippocampal interneurons and hilar cells in the dentate gyrus.

\section{Lyc improves radiation-induced behavioral impairments}

Our data from open field, forced swimming and tail suspension tests have shown that mice irradiated with 5.5 Gy suffered less locomotor activity and depression. These results confirmed that the radiation dosage we applied was sufficient to induce the neurobehavioral impairments of the exposed mice(Wang et al., 2019b). Morris water maze test has suggested the radiation induced spatial cognitive dysfunction(Schoenfeld et al., 2017). Four-week oral administration of Lyc could effectively prevent those radiation induced behavioral and cognitive impairments. It 
has been reported that LBP could significantly improve the dextromethorphaninduced depression in the rat model(Po et al., 2017). In the APP/PS1 double transgenic mice, a widely used animal model of Alzheimer's disease, 2-week oral administration of Lyc significantly improved their spatial memory in Morris water maze test(Zhang et al., 2013). This radioprotective effect of Lyc should be related to the previously suggested effects of Lyc or LBP, including anti-oxidation, anti-inflammation, preventing neuron loss and promoting neuronal regeneration(Xing et al., 2016). The anti-oxidative effect of Lyc or LBP should be due to their activities in scavenging reactive oxygen species (ROS) and inhibiting oxidases(Hsieh et al., 2018; Liu et al., 2020; Wang et al., 2017). By using primary cultured neonatal rat hippocampal neurons, LBP significantly decreased the overexpression of inflammatory cytokines and apoptotic biomarkers induced by oxygen-glucose deprivation/reperfusion(Zhao et al., 2017). These anti-oxidative and anti-inflammatory effects of Lyc should contribute to its neuroprotection and subsequent improvement of spatial memory and depression.

\section{Lyc improves radiation-induced hippocampal neuron loss}

Our previous study has demonstrated that the oral administration of epimedium (Wang et al., 2019b) or intraperitoneal injection of amitriptyline, an inhibitor of acid sphingomyelinase (ASMase) (Guo et al., 2019) significantly prevent radiation-induced loss of Ki67, doublecortin (DCX) and PV immunopositive cells in the subgranular zone (SGZ) of the dentate gyrus, indicating the improvement of neurogenesis in SGZ. In the pilocarpine induced mouse model of status epilepticus, dramatic reduction of NeuN, CB and PV immunopositive neurons in different hippocampal areas implicated that these biomarkers should help to present the status of neuron loss and neuron regeneration in hippocampus(Xu and Tang, 2018; Zhang et al., 2009). A recent study has reported that LBP treatment could increase the number of NeuN positive neurons in the dentate gyrus of APP/PS1 mice(Zhou et al., 2020). In this study, our behavioral tests have demonstrated obvious cognitive impairment and depression in those irradiated mice. Therefore, we focused on the changes of those 
hippocampal neurons and interneurons which should related to the cognitive dysfunction and depression.

The number of NeuN-immunopositive neurons in the dentate hilus could be reduced by ionizing irradiation(Pipová Kokošová et al., 2020). In the chronic mouse model of temporal lobe epilepsy (TLE), reduced number of the hilar mossy cells was found to be the major reason of spatial memory deficit(Bui et al., 2018). As the major excitatory neurons in dentate hilus, mossy cells are responsible for receiving inputs from local interneurons, granule cells and the CA3 input and protruding axons into different layers of the dentate gyrus(GoodSmith et al., 2017; Houser et al., 2021). They play essential roles in maintaining cognitive neuronal network and even in the regeneration and/or the reorganization of neuronal network after neuronal damage(Marqués-Marí et al., 2007; Oh et al., 2020; Zhang et al., 2009). Therefore, the decreased number of hilar NeuN immunopositive cells after irradiation in this study may implicate the mossy cell loss and the consequent cognitive deficit and depression(Oh et al., 2020).

Several previous studies have demonstrated that the loss of hippocampal interneurons should lead to cognitive dysfunction and behavior alteration(Adotevi and Leitch, 2017; Ma and McLaurin, 2014; Takahashi et al., 2010). As a calcium binding protein expressed in some hippocampal interneurons, CB plays a critical role in hippocampal learning function(Li et al., 2017b). Down-regulated hippocampal CB expression and/or CB positive neurons were observed in various neurological disorders and were related to cognitive deficits(Goffigan-Holmes et al., 2019; Li et al., 2017a; You et al., 2017), while the increased number of $C B$ positive hippocampal neurons implicated the generation of new neurons and the establishment of functional neuronal network(Ramirez-Rodriguez et al., 2019). The CB positive interneurons located in the strata radiatum, lacunosum moleculare (CA1-SRLM) and oriens (CA1-SO) of CA1 area are mostly GABAergic interneurons which mainly form synapses with CA1 pyramidal cells and are significantly reduced during ageing(Gulyás and Freund, 1996; 
Potier et al., 1994). In the present study, we found that the radiation exposure reduced the number of $\mathrm{CB}$ positive interneurons in the CA1-SRLM, which are involved in the regulation of the inputs from the entorhinal cortex to hippocampal CA1 apical dendrites of pyramidal neurons or spatial information(Li et al., 2017b). And the reduced number of $\mathrm{CB}$ expressing interneurons in the CA1-SO may affect the interaction between the CA1 pyramidal cells and CA1-projecting subiculum neurons which should play a role in the spatial memory(Blasco-Ibáñez and Freund, 1995; Sun et al., 2019).

PV positive cells in hippocampal area are inhibitory GABAergic interneurons which function to configure learning related neuronal network(Donato et al., 2013). The PV positive interneurons in CA1 area provide inhibitory synapses onto CA1 pyramidal neurons to regulate their activities in cognitive network(Udakis et al., 2020; Yi et al., 2014). Promotion of PV immunopositive interneurons in CA1 area could rescue cognitive dysfunction of adult mouse model of schizophrenia(Mukherjee et al., 2019). In addition, the activity of PV positive interneurons in dentate gyrus should have an essential role in cognitive function(Elgueta and Bartos, 2019; Medrihan et al., 2020). The irradiation-induced PV immunopositive neuron loss in the stratum pyramidum of CA1 (CA1-SP) and in the stratum granulosum of the dentate gyrus (DG-SG) was observed in current study, which should be responsible for the cognitive deficits of those irradiated mice.

The Lyc has been reported as an anti-depressive drug previously(Zhang et al., 2012). Our behavioral tests indicated the spatial memory impairment and depression of those radiation exposure mice. Given these, we proposed that the radiation exposure at the dosage of $5.5 \mathrm{~Gy}$ induced the depressive behavior and the spatial memory impairment of mice by promoting the hippocampal NeuN-positive neuron loss in the hilus, CB-positive interneuron loss in the CA1-SO and CA1-SRLM, and the PV-positive interneuron loss in the CA1-SP and DG-SG areas. Oral administration of Lyc significantly prevented the neuron loss in these areas and then maintained the 
hippocampal neuronal network which consequently improved the radiation induced cognitive deficit and depression.

\section{Conclusion}

Ionizing radiation at the dosage of 5.5 Gy induced the depressive behavior and spatial memory impairment in BALB/c mice 4 weeks after irradiation. Significant NeuN-positive neuron loss in the hilus of the dentate gyrus, CB-positive interneuron loss in the CA1-SO and CA1-SRLM, and PV-positive interneuron loss in the CA1-SP and DG-SG areas 6 weeks after irradiation were demonstrated by immunohistochemistry study. Oral administration of Lyc prevented the loss of those hippocampal neurons and then improved the radiation-induced cognitive dysfunction and depression. While further study is still needed to elucidate the molecular mechanism of Lyc, current study strongly suggests that this "superfood" may be a promising radio-neuro-protective supplement to prevent radiotherapy or other ionizing radiation induced cognitive impairment.

\section{Ethics approval}

The research protocols involving BALB/c mice in this study were reviewed and approved by the Ethics Committee of Yangtze University Health Science Center.

\section{Consent for publication}

Not applicable.

\section{Availability of data and materials}

All data generated or analysed during this study are included in this article.

\section{Competing interests}

The authors declare that they have no competing interests 


\section{Funding}

This work was supported by grants from the College Students Innovative Entrepreneurial Training Program of Hubei province (S20191049071) to GL, the Scientific Research Foundation of Hubei Health Commission (WJ2016-Y-01), the Jingzhou Science and Technology Department (2019EC61-17) and the Yangtze Fund for Youth Teams of Science and Technology Innovation (2016cqt04) to QF, the nature science foundation of Hubei province (2017CFB786) and the Jingzhou Science and Technology Department (2017-93) to PXC, the Research Project of Traditional Chinese Medicine (WJ2017-15) and the Scientific Research Foundation of Hubei Health Commission (WJ2019-02) to HJR, and National Research Foundation of Singapore to Singapore Nuclear Research and Safety Initiative (TFR).

\section{Authors' contributions}

Lei Guo, Qian-Qian Du and Piao-Qin Cheng: Investigation. Ting-Ting Yang,

Chao-Qun Xing and Xue-Zhi Luo: Formal analysis. Xiao-Chun Peng: Resources, Methodology. Feng Qian: Conceptualization, Data Curation, Writing - Original Draft. Jiang-Rong Huang: Project administration, Feng-Ru Tang: Conceptualization, Methodology, Writing - Review \& Editing.

\section{Acknowledgments}

Not applicable.

\section{References}

Adotevi, N.K., Leitch, B., 2017. Synaptic Changes in AMPA Receptor Subunit Expression in Cortical Parvalbumin Interneurons in the Stargazer Model of Absence Epilepsy. Front Mol Neurosci 10.

Allen, B.D., Acharya, M.M., Lu, C., Giedzinski, E., Chmielewski, N.N., Quach, D., Hefferan, M., Johe, K.K., Limoli, C.L., 2018. Remediation of Radiation-Induced Cognitive Dysfunction through Oral Administration of the Neuroprotective Compound NSI-189. Radiat Res 189, 345-353. 
Blasco-Ibáñez, J.M., Freund, T.F., 1995. Synaptic input of horizontal interneurons in stratum oriens of the hippocampal CA1 subfield: structural basis of feed-back activation. Eur J Neurosci 7, 2170-2180.

Bui, A.D., Nguyen, T.M., Limouse, C., Kim, H.K., Szabo, G.G., Felong, S., Maroso, M., Soltesz, I., 2018. Dentate gyrus mossy cells control spontaneous convulsive seizures and spatial memory. Science 359, 787-790.

de Souza Zanchet, M.Z., Nardi, G.M., de Oliveira Souza Bratti, L., Filippin-Monteiro, F.B., Locatelli, C., 2017. Lycium barbarum Reduces Abdominal Fat and Improves Lipid Profile and Antioxidant Status in Patients with Metabolic Syndrome. Oxid Med Cell Longev 2017, 9763210.

Donato, F., Rompani, S.B., Caroni, P., 2013. Parvalbumin-expressing basket-cell network plasticity induced by experience regulates adult learning. Nature 504, 272-+. Elgueta, C., Bartos, M., 2019. Dendritic inhibition differentially regulates excitability of dentate gyrus parvalbumin-expressing interneurons and granule cells. Nat Commun 10,5561 .

Goffigan-Holmes, J., Sanabria, D., Diaz, J., Flock, D., Chavez-Valdez, R., 2019.

Calbindin-1 Expression in the Hippocampus following Neonatal Hypoxia-Ischemia and Therapeutic Hypothermia and Deficits in Spatial Memory. Dev Neurosci, 1-15. GoodSmith, D., Chen, X., Wang, C., Kim, S.H., Song, H., Burgalossi, A., Christian, K.M., Knierim, J.J., 2017. Spatial Representations of Granule Cells and Mossy Cells of the Dentate Gyrus. Neuron 93, 677-690.e675.

Gulyás, A.I., Freund, T.F., 1996. Pyramidal cell dendrites are the primary targets of calbindin D28k-immunoreactive interneurons in the hippocampus. Hippocampus 6, 525-534.

Guo, Y.R., Liu, Z.W., Peng, S., Duan, M.Y., Feng, J.W., Wang, W.F., Xu, Y.H., Tang, X., Zhang, X.Z., Ren, B.X., Tang, F.R., 2019. The Neuroprotective Effect of Amitriptyline on Radiation-Induced Impairment of Hippocampal Neurogenesis. Dose Response 17, 1559325819895912.

Hladik, D., Tapio, S., 2016. Effects of ionizing radiation on the mammalian brain. Mutat Res 770, 219-230.

Houser, C.R., Peng, Z., Wei, X., Huang, C.S., Mody, I., 2021. Mossy Cells in the Dorsal and Ventral Dentate Gyrus Differ in Their Patterns of Axonal Projections. J Neurosci 41, 991-1004.

Hsieh, F.C., Hung, C.T., Cheng, K.C., Wu, C.Y., Chen, Y.C., Wu, Y.J., Liu, W.T., Chiu, C.C., 2018. Protective Effects of Lycium barbarism Extracts on UVB-Induced Damage in Human Retinal Pigment Epithelial Cells Accompanied by Attenuating ROS and DNA Damage. Oxidative Medicine and Cellular Longevity 2018. Jacob, J., Durand, T., Feuvret, L., Mazeron, J.J., Delattre, J.Y., Hoang-Xuan, K., Psimaras, D., Douzane, H., Ribeiro, M., Capelle, L., Carpentier, A., Ricard, D., Maingon, P., 2018. Cognitive impairment and morphological changes after radiation therapy in brain tumors: A review. Radiother Oncol 128, 221-228.

Lee, S.C., Wang, T.J., Chu, P.Y., 2019. Predictors of weight loss during and after radiotherapy in patients with head and neck cancer: A longitudinal study. European Journal of Oncology Nursing 39, 98-104.

Li, H.Y., Huang, M., Luo, Q.Y., Hong, X., Ramakrishna, S., So, K.F., 2019. Lycium barbarum (Wolfberry) Increases Retinal Ganglion Cell Survival and Affects both Microglia/Macrophage Polarization and Autophagy after Rat Partial Optic Nerve Transection. Cell Transplant 28, 607-618.

Li, J.T., Xie, X.M., Yu, J.Y., Sun, Y.X., Liao, X.M., Wang, X.X., Su, Y.A., Liu, Y.J., Schmidt, M.V., Wang, X.D., Si, T.M., 2017a. Suppressed Calbindin Levels in 
Hippocampal Excitatory Neurons Mediate Stress-Induced Memory Loss. Cell Rep 21, 891-900.

Li, Y.D., Xu, J.M., Liu, Y.F., Zhu, J., Liu, N., Zeng, W.B., Huang, N., Rasch, M.J., Jiang, H.F., Gu, X., Li, X., Luo, M.H., Li, C.Y., Teng, J.L., Chen, J.G., Zeng, S.Q., Lin, L.N., Zhang, X.H., 2017b. A distinct entorhinal cortex to hippocampal CA1 direct circuit for olfactory associative learning. Nat Neurosci 20, 559-+.

Liu, L., Sha, X.Y., Wu, Y.N., Chen, M.T., Zhong, J.X., 2020. Lycium barbarum polysaccharides protects retinal ganglion cells against oxidative stress injury. Neural Regeneration Research 15, 1526-1531.

Ma, K., McLaurin, J., 2014. alpha-Melanocyte Stimulating Hormone Prevents GABAergic Neuronal Loss and Improves Cognitive Function in Alzheimer's Disease. J Neurosci 34, 6736-6745.

Ma, Z.F., Zhang, H., Teh, S.S., Wang, C.W., Zhang, Y., Hayford, F., Wang, L., Ma, T., Dong, Z., Zhu, Y., 2019. Goji Berries as a Potential Natural Antioxidant Medicine: An Insight into Their Molecular Mechanisms of Action. Oxid Med Cell Longev 2019, 2437397.

Marqués-Marí, A.I., Nacher, J., Crespo, C., Gutièrrez-Mecinas, M., Martínez-Guijarro, F.J., Blasco-Ibáñez, J.M., 2007. Loss of input from the mossy cells blocks maturation of newly generated granule cells. Hippocampus 17, 510-524.

Medrihan, L., Umschweif, G., Sinha, A., Reed, S., Lee, J., Gindinova, K., Sinha, S.C., Greengard, P., Sagi, Y., 2020. Reduced Kv3.1 Activity in Dentate Gyrus Parvalbumin Cells Induces Vulnerability to Depression. Biol Psychiatry 88, 405-414.

Mukherjee, A., Carvalho, F., Eliez, S., Caroni, P., 2019. Long-Lasting Rescue of Network and Cognitive Dysfunction in a Genetic Schizophrenia Model. Cell 178, 1387-+.

Oh, S.J., Cheng, J., Jang, J.H., Arace, J., Jeong, M., Shin, C.H., Park, J., Jin, J., Greengard, P., Oh, Y.S., 2020. Hippocampal mossy cell involvement in behavioral and neurogenic responses to chronic antidepressant treatment. Mol Psychiatry 25, 1215-1228.

Pipová Kokošová, N., Kisková, T., Vilhanová, K., Štafuriková, A., Jendželovský, R., Račeková, E., Šmajda, B., 2020. Melatonin mitigates hippocampal and cognitive impairments caused by prenatal irradiation. Eur J Neurosci 52, 3575-3594.

Po, K.K.T., Leung, J.W.H., Chan, J.N.M., Fung, T.K.H., Sanchez-Vidana, D.I., Sin, E.L.L., So, K.F., Lau, B.W.M., Siu, A.M.H., 2017. Protective effect of Lycium Barbarum polysaccharides on dextromethorphan-induced mood impairment and neurogenesis suppression. Brain Research Bulletin 134, 10-17.

Potier, B., Krzywkowski, P., Lamour, Y., Dutar, P., 1994. Loss of calbindin-immunoreactivity in CA1 hippocampal stratum radiatum and stratum lacunosum-moleculare interneurons in the aged rat. Brain Res 661, 181-188.

Ramirez-Rodriguez, G.B., Olvera-Hernandez, S., Vega-Rivera, N.M., Ortiz-Lopez, L., 2019. Melatonin Influences Structural Plasticity in the Axons of Granule Cells in the Dentate Gyrus of Balb/C Mice. Int J Mol Sci 20.

Schoenfeld, R., Schiffelholz, T., Beyer, C., Leplow, B., Foreman, N., 2017. Variants of the Morris water maze task to comparatively assess human and rodent place navigation. Neurobiology of Learning and Memory 139, 117-127.

Sun, Y., Jin, S., Lin, X., Chen, L., Qiao, X., Jiang, L., Zhou, P., Johnston, K.G., Golshani, P., Nie, Q., Holmes, T.C., Nitz, D.A., Xu, X., 2019. CA1-projecting subiculum neurons facilitate object-place learning. Nat Neurosci 22, 1857-1870.

Takahashi, H., Brasnjevic, I., Rutten, B.P.F., Van Der Kolk, N., Perl, D.P., Bouras, C., Steinbusch, H.W.M., Schmitz, C., Hof, P.R., Dickstein, D.L., 2010. Hippocampal 
interneuron loss in an APP/PS1 double mutant mouse and in Alzheimer's disease. Brain Struct Funct 214, 145-160.

Tang, F.R., Loke, W.K., Khoo, B.C., 2017. Postnatal irradiation-induced hippocampal neuropathology, cognitive impairment and aging. Brain Dev 39, 277-293.

Tian, X., Liang, T., Liu, Y., Ding, G., Zhang, F., Ma, Z., 2019. Extraction, Structural Characterization, and Biological Functions of Lycium Barbarum Polysaccharides: A Review. Biomolecules 9.

Udakis, M., Pedrosa, V., Chamberlain, S.E.L., Clopath, C., Mellor, J.R., 2020. Interneuron-specific plasticity at parvalbumin and somatostatin inhibitory synapses onto CA1 pyramidal neurons shapes hippocampal output. Nat Commun 11, 4395. Ulbricht, C., Bryan, J.K., Costa, D., Culwell, S., Giese, N., Isaac, R., Nummy, K., Pham, T., Rapp, C., Rusie, E., Weissner, W., Windsor, R.C., Woods, J., Zhou, S., 2014. An Evidence-Based Systematic Review of Goji (Lycium spp.) by the Natural Standard Research Collaboration. J Diet Suppl 12, 184-240.

Wang, J., Yao, Y., Liu, X., Wang, K., Zhou, Q., Tang, Y., 2019a. Protective effects of lycium barbarum polysaccharides on blood-retinal barrier via ROCK1 pathway in diabetic rats. Am J Transl Res 11, 6304-6315.

Wang, S.W., Ren, B.X., Qian, F., Luo, X.Z., Tang, X., Peng, X.C., Huang, J.R., Tang, F.R., 2019b. Radioprotective effect of epimedium on neurogenesis and cognition after acute radiation exposure. Neuroscience Research 145, 46-53.

Wang, Y.Y., Ding, L., Li, Y.M., Guan, C.R., Guo, J., 2017. Lycium barbarum polysaccharides can reduce the oxidative damage of the retinal nerve cells in diabetic rats. International Journal of Clinical and Experimental Medicine 10, 5168-5174.

Xing, X.W., Liu, F.Y., Xiao, J., So, K.F., 2016. Neuro-protective Mechanisms of Lycium barbarum. Neuromolecular Medicine 18, 253-263.

Xu, J.H., Tang, F.R., 2018. Voltage-Dependent Calcium Channels, Calcium Binding Proteins, and Their Interaction in the Pathological Process of Epilepsy. Int J Mol Sci 19.

Yang, D., So, K.F., Lo, A.C., 2017. Lycium barbarum polysaccharide extracts preserve retinal function and attenuate inner retinal neuronal damage in a mouse model of transient retinal ischaemia. Clin Exp Ophthalmol 45, 717-729.

Yi, F., Ball, J., Stoll, K.E., Satpute, V.C., Mitchell, S.M., Pauli, J.L., Holloway, B.B., Johnston, A.D., Nathanson, N.M., Deisseroth, K., Gerber, D.J., Tonegawa, S., Lawrence, J.J., 2014. Direct excitation of parvalbumin-positive interneurons by M1 muscarinic acetylcholine receptors: roles in cellular excitability, inhibitory transmission and cognition. J Physiol 592, 3463-3494.

You, J.C., Muralidharan, K., Park, J.W., Petrof, I., Pyfer, M.S., Corbett, B.F., LaFrancois, J.J., Zheng, Y., Zhang, X.H., Mohila, C.A., Yoshor, D., Rissman, R.A., Nestler, E.J., Scharfman, H.E., Chin, J., 2017. Epigenetic suppression of hippocampal calbindin-D28k by Delta FosB drives seizure-related cognitive deficits. Nat Med 23, 1377-+.

Zhang, E., Yau, S.Y., Lau, B.W., Ma, H., Lee, T.M., Chang, R.C., So, K.F., 2012. Synaptic plasticity, but not hippocampal neurogenesis, mediated the counteractive effect of wolfberry on depression in rats(1). Cell Transplant 21, 2635-2649.

Zhang, Q.L., Du, X.P., Xu, Y.P., Dang, L., Xiang, L., Zhang, J.W., 2013. The effects of Gouqi extracts on Morris maze learning in the APP/PS1 double transgenic mouse model of Alzheimer's disease. Experimental and Therapeutic Medicine 5, 1528-1530. Zhang, S., Khanna, S., Tang, F.R., 2009. Patterns of Hippocampal Neuronal Loss and Axon Reorganization of the Dentate Gyrus in the Mouse Pilocarpine Model of Temporal Lobe Epilepsy. J Neurosci Res 87, 1135-1149. 
Zhao, P., Ma, N.T., Chang, R.Y., Li, Y.X., Hao, Y.J., Yang, W.L., Zheng, J., Niu, Y., Sun, T., Yu, J.Q., 2017. Mechanism of Lycium barbarum polysaccharides on primary cultured rat hippocampal neurons. Cell and Tissue Research 369, 455-465.

Zhou, Y., Duan, Y., Huang, S., Zhou, X., Zhou, L., Hu, T., Yang, Y., Lu, J., Ding, K., Guo, D., Cao, X., Pei, G., 2020. Polysaccharides from Lycium barbarum ameliorate amyloid pathology and cognitive functions in APP/PS1 transgenic mice. Int J Biol Macromol 144, 1004-1012. 